\title{
Editorial
}

\section{La Cultura de los Cuidados y las Instituciones de Salud en Nuestros Tiempos en Latinoamérica}

\author{
Dinora Rebolledo Malpica \\ Docente titular de la Universidad "Lisandro Alvarado" de Barquisimeto Venezuela \\ Dra. Por la Universidad de Alicante (Programa: Enfermería Y Cultura de los Cuidados"). \\ Cómo citar esta editorial en edición digital: Rebolledo Malpica, D. (2013) La Cultura de los Cuidados y las Instituciones de \\ Salud en Nuestros Tiempos en Latinoamérica. Cultura de los Cuidados (Edición digital) 17, 37. \\ Disponible en: <http://dx.doi.org/10.7184/cuid.2013.37.01> \\ Correspondencia: Avenida las Palmas con Avenida Andrés Bello Decanato de Ciencias de la Salud. \\ Código Postal 3001, Barquisimeto (Venezuela). Correo electrónico: dinorarebolledo@ucla.edu.ve
}



La organización de los cuidados de salud institucionalizados especialmente en la Latinoamérica gira alrededor de sistemas políticos y sociales complejos, que muchas veces dejan de lado la verdadera razón de ser y existencia de tales empresas sanitarias: El paciente.

Hasta hace poco, según el modelo clásico y tradicional de las organizaciones de sanitarias, el paradigma de referencia en la atención en salud especialmente en occidente, se basaba exclusivamente en un modelo centrado en el médico y en la medicina, disciplina dominante y hegemónica en la organización de cuidados de salud de la región. Este predomino sobre la organización de los cuidados en nuestra cultura sanitaria, llevo a planear la atención del paciente basándose en la patología del mismo. Es decir, con un enfoque del cuidado centrado en patología medicas; en el cual los pacientes son agrupados y distribuidos en es un espacio físico y clasificados por enfermedad, que le permite, según esta concepción de cuidados, obtener de ellos el máximo tiempo y el mínimo esfuerzo del equipo de salud o como lo dice Fourcault (2009) "mantenerlos es una visibilidad sin lagunas... y construir sobre ellos un saber que se centraliza"

Esta forma de organización de la atención, sin considerar otro criterio de agrupación, deja el entorno biopsicosocial del paciente fuera del contexto sanitario y lleva al cuidado a convertirse en una sencilla actividad de atención médica, haciendo un reduccionismo simple y vació del cuidado a una sola perspectiva de salud y al paciente lo disminuye a "una patología médica" o dicho de otra manera a un mero "objeto".

Por otro lado, la cultura imperante en estos centros asistenciales es hermética y cerrada para el paciente, y se convierte en un factor 
aislante del mismo. Es sabido por todos los del medio sanitario, que al ser ingresado en una institución hospitalaria, (especialmente en Latinoamérica debido a cierto sentimiento de desconfianza que el colectivo siente hacia las instituciones del estado, entre otros) el paciente se siente indefenso ya que ve interrumpida su cotidianidad, sus hábitos y sus costumbres, para adentrase en un mundo desconocido y a veces temido. El paciente puede llegar a sentir el ingreso al centro hospitalario como un estado de "confinamiento del ser humano" con la consecuente perdida de libertad, donde asume nuevos códigos de comportamiento. Asimismo, la jerga empleados por los profesionales de salud, así como la burocratización de los procedimientos, es decir, la cultura organizacional de las instituciones de salud, provoca en el paciente un sentimiento soledad, desconcierto y abandono, que lo lleva a una situación vulnerable y desconocida para él. Esta paradoja se ve en la separación visible que existe entre los cuidados satisfactorios y las estructuras sanitarias de nuestros tiempos.

Es importante considerar que las respuestas que el paciente tiene ante el padecimiento o enfermedad, no son solo de la patología de base, es decir, no son solo fisiopatológicas o biológicas. El paciente es un ser cultural que vive en un entorno, en una familia, y que posee sus propias estructuras sociales y culturales que condicionan su respuesta a la enfermedad; y muchas veces, estas estructuras culturales se ven desplazadas por la institucionalización de los cuidados de salud. Los pacientes deben ser vistos como un ser integral, biopsicosocial y holístico que requiere cuidados culturalmente competentes.

Por otra lado, para enfermería el cuidado es su objeto epistémico, los avances en la percepción tanto del fundamento de la práctica de enfermería, como de su propio entorno social, y sobre todo del desarrollo de su objeto disciplinar, le ha permitido una visión ampliada de su actuación y desempeño dentro de las instituciones de salud, reconociendo su aporte al cumplimiento de los objetivos de las mismas, así como su contribución en la satisfacción de las necesidades del paciente: eje central de la existencia de dichas instituciones. Enfermería comparte con las organizaciones de salud, su objeto epistémico y de existencia, ya que mientras estas empresas existen para satisfacer las necesidades de salud de los pacientes, enfermería tiene claro que su objeto disciplinar es proporcionar un cuidado satisfactorio a esos mismos pacientes.

Bajo esta misma perspectiva, la visión del paciente como ser biopsicosocial y holístico lleva a replantear la necesidad de un cambio paradigmático en la organización de los cuidados de salud en los centros asistenciales de nuestro entorno, sobre todo con el advenimiento de los cuidados culturales, disciplina ampliamente desarrollada también desde enfermería. Los sistemas sanitarios y especialmente los profesionales de la salud, están siendo cada día mas sensibilizados a cambiar esa concepción estereotipada de la atención de salud centrada en el médico y en la medicina y los ha llevado a proponer unos cuidados centrados en el paciente.

Asimismo, el sistema sanitario de la región está cada día mas interesado en descubrir los puntos de vista émicos, personales o culturales, relativos a los cuidados. Todavía queda mucho por andar sobre esta conducta en nuestros hospitales, sin embargo, con los avances académicos y tecnológicos de las disciplina, se ha despertado una conciencia de que no se puede proporcionar cuidados al paciente de manera aislada de su contexto y que este es un 
protagonista activo del proceso de cuidado, que cada día exige mas, un papel relevante en tal proceso

Hoy por hoy y bajo el auspicio de la globalización y la masificación de los medios de comunicación, no se puede seguir considerando al paciente como una caja vacía, que viene a nuestros encuentro solo para recibir, y recibir atención, sin preguntar o sin que se vea involucrado en la misma. Esta concepción del paciente como agente pasivo queda descontextualizada en las nuevas empresas sanitarias, enfocada en los cuidados culturales, ya que el paciente es un protagonista activo y participativo de su propia proceso salud-enfermedad, y pide que se reconozcan sus los valores, creencias y prácticas que están relacionadas con la salud y con el cuidado que ellos mismos se realizan, haciendo cada día más exigente y culturalmente competente el proporcionar los cuidados adecuado a este paciente, dentro de las instituciones de salud.

Al convertirse en un actor protagonista, el paciente espera recibir de los centros asistenciales a los que acude, una atención centrada en su contexto y cotidianidad de vida, es decir una atención individualizada, concentrada específicamente en su situación de salud actual pero con un enfoque holístico, sin dejar por fuera su contexto social, familiar y cultural. Solo bajo este enfoque los profesionales de enfermería están en condiciones de proporcionar un cuidado satisfactorio y congruente con las verdaderas necesidades del paciente. Por lo tanto, la organización de los cuidados institucionalizados debe estar centrada en los cuidados culturales. Esto último se observa en la aprobación de los enfoques psicó - socio - culturales que en los últimos años se han extendido en los estudios e investigaciones desarrollados en las organizaciones salud de la zona.
Este cambio paradigmático en los enfoques del cuidado, ha obligado a las instituciones de salud a cambiar sus políticas de atención, haciendo que cada día mas, se tome en cuenta el contexto cultural de los paciente, garantía que permite cuidados mas satisfactorios, esto se ve reflejado en las diferentes disciplina que convergen en los centros asistenciales, ya que en sus pensum de estudio se nota el cuidado cultural como una asignatura de curso. También se nota que en algunos centros se le permite al paciente mantener su rutina de culto religioso, con normas de visitas de los lideres religiosos elegidos por el paciente, se le permite tomar sus propias decisiones sobre su cuidados, tal es el caso de los testigos de Jehová; Se les permite un acompañamiento de familiares, como el caso de madres de recién nacidos prematuros, las salas de neonatología tienen espacio para que las madres pernoten en ellas, lo mas cerca de sus hijos, e incluso se han habilitados salas de espera acondicionados para los familiares, y durante los cuidados de salud se involucra al familiar en los mismo, se le educa sobre lo cuidados mínimos, de tal manera que no se alejen del entorno del paciente, esto contribuye a la estabilidad social, cultural y emocional del paciente durante su recuperación. Por otro lado, se le permite al paciente traer a su unidad de hospitalización sus objetos personales, para crear un ambiente lo mas parecido posible a su propio entono familiar y por ultimo, pero muy importante, que por encima de todo se cuida la identidad personal, al no ser sustituida ésta por un número de cama o por el nombre de una patología.

Por otro lado, cada día existen más publicaciones científicas referidas a la sensibilización de los cuidados culturales en los profesionales de la salud, que vienen de otros espacios geográficos y de la región misma. Entre los de 
afuera, cabe destacar la revista de Cultura de los Cuidados, editada por la universidad de Alicante. Es decir, que se ha despertando una conciencia de hacer de la atención de salud un cuidado cultural que permita el reconocimiento del otro, respetando las experiencias, conocimientos y actitudes que el paciente trae consigo al momento de entrar a los centros de salud.

Esta conciencia ha llevado a la comunidad científica que investiga sobre los cuidados a crear una plataforma teórica y científica sólida del cuidado cultural, tal cual es el caso de Madeleine Leininger, Rachel Spector, Larry Purnell y desde España, José Siles, entre otros, quienes se han dedicado en los últimos años a mostrar como el objeto epistémico de enfermería se fortalece desde la visión de los cuidados culturales. En Latinoamérica aun queda mucho por andar, sin embargo, se evidencia, como ya se dijo, el despertar de la conciencia de los cuidados culturalmente competente.

Bajo esta perspectiva, se puede decir que los modelos de organizaciones de los centros asistenciales de nuestros tiempos y de la región latinoamericana están pasando por un momento de transición cultural, que le permite centrar la atención en el paciente con un enfoque cultural más que en disciplinas hegemónicas, profesionales de salud o asuntos burocráticos. Permitiendo reconocer el valor en el otro y llegar a saber qué prácticas podrían preservar, cuáles negociar y cuales reestructurar, con el fin último de proporcionar unos cuidados culturalmente satisfactorios.

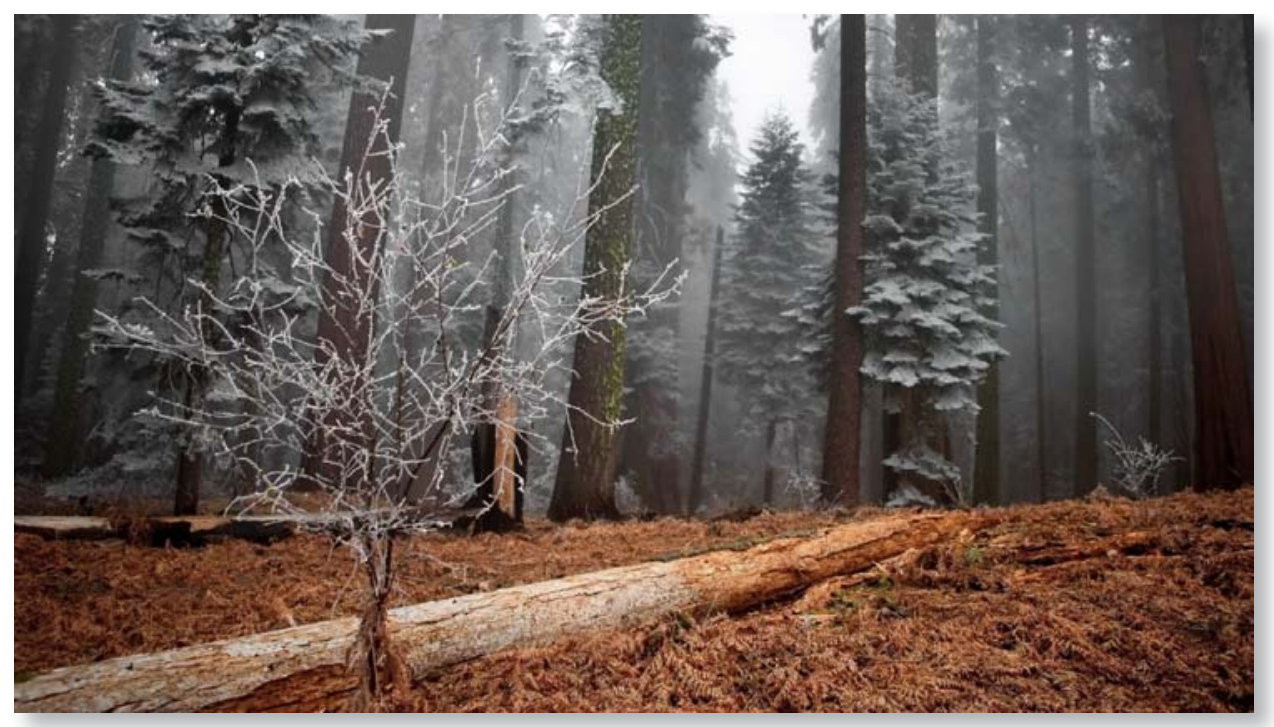

